\title{
A Case of Hodgkin Lymphoma With Polyarthralgia After Completion of Chemotherapy
}

\author{
Emre ATA, Selim AKARSU, Levent TEKİN \\ Department of Physical Medicine and Rehabilitation, Gülhane Military Medical Academy \\ Haydarpaşa Training Hospital, İstanbul, Turkey
}

There are various musculoskeletal manifestations that may develop in a patient after chemotherapy. These manifestations may be due to metastasis to musculoskeletal structures, paraneoplastic syndrome or immune reactions as well as adverse reactions to cancer specific chemotherapy. ${ }^{1}$ One of these symptoms is post-chemotherapy rheumatism which is a non-inflammatory and selflimiting condition that manifests as symmetric or asymmetric arthralgia, arthritis and stiffness that involves both large and small joints. ${ }^{2}$ It has been described in patients with some types of cancer including breast cancer, ovarian cancer, and nonHodgkin's lymphoma within a few months after the completion of chemotherapy. ${ }^{2}$ In this article, we report a patient with polyarthralgia after chemotherapy for Hodgkin lymphoma.

A 39-year-old male patient presented with a six-week history of symmetric arthralgia on his bilateral hand and foot joints. Roughly eight months prior to these symptoms, he had Hodgkin lymphoma diagnosis and was treated with chemotherapy, including palonosetron, doxorubicin, cyclophosphamide, etoposide, and vincristine. His last chemotherapy session was six weeks prior to the onset of arthralgia. Physical examination revealed no swelling on any joints but there was tenderness on bilateral hand and foot joints with palpation (at the proximal interphalangeal, metacarpophalangeal, ankle and metatarsophalangeal joints). There was no synovitis on ultrasonographic joint evaluation. He had no history of any other systemic disease and no family history of rheumatic disease.

Laboratory results were as follows: erythrocyte sedimentation rate $65 \mathrm{~mm} /$ hour, C-reactive protein $41 \mathrm{mg} / \mathrm{L}$, negative results for rheumatoid factor, anti-cyclic citrullinated peptide antibodies, antinuclear antibodies, ribonucleoprotein antibodies, anti-Sjögren's syndrome A, anti-Sjögren's syndrome $\mathrm{B}$, anti-systemic sclerosis topoisomerase I, anti-jo1, anti-double stranded deoxyribonucleic acid and anti-histone antibodies. Other laboratory findings were unremarkable. Oral acemetacin $60 \mathrm{mg}$ twice-a-day was commenced and continued for three weeks. Inflammatory markers and articular symptoms improved after treatment.

In conclusion, post-chemotherapy rheumatism may be seen after completion of chemotherapy in patients with Hodgkin lymphoma. Clinicians should keep this possibility in mind to speed up the diagnosis process without unnecessary investigation and it would be advisable if the patients are informed about this complication before chemotherapy treatment for relieving their concerns. 


\section{Declaration of conflicting interests}

The authors declared no conflicts of interest with respect to the authorship and/or publication of this article.

\section{Funding}

The authors received no financial support for the research and/or authorship of this article.

\section{REFERENCES}

1. Leandro MJ, Isenberg DA. Rheumatic diseases and malignancy--is there an association? Scand $\mathrm{J}$ Rheumatol 2001;30:185-8.

2. Amiri AH, Rafiei A. Analysis of patients with postchemotherapy arthralgia and arthritis in breast cancer. Indian J Med Sci 2010;64:197-203. 